\title{
El estudio de casos en las bases de datos del Science Citation Index, Social Science Citation Index y Arts and Humanities Citation Index (1992-2000)
}

\section{Emilio Delgado López-Cózar y Antonio Fernández Cano}

Arbor CLXXI, 675 (Marzo), 609-629 pp.

Este estudio bibliométrico indaga el status de la metodología del estudio de casos, utilizando intercambiablemente los términos CASE STUDY y CASE-REPORT, en las bases del INSTITUTE FOR SCIENTIFIC INFORMATION (ISI) de Filadelfia: SCIENCE Citation INDEX (SCI), Social SCIENCE Citation INDEX (SSCI) y ARTS AND HUMANITIES CITATION INDEX (A\&HCI).

El hallazgo central testimonia un uso extenso, progresivo y diversificado del estudio de caso en tales bases, a lo largo del tiempo, en las más diversas disciplinas, temáticas y en un gran número de revistas científicas.

\section{Introducción}

Dentro del repertorio de métodos de investigación disponibles contamos con el Estudio de Casos [desde ahora abreviadamente EC]; tal vez sea éste el método primario de indagación científica. No vamos a discutir aquí el estatus metodológico del EC (véanse aportes y discusión abundante en este mismo número), sino que trataremos de averiguar cómo y cuánto se está utilizando este método en los diversos ámbitos del conocimiento a escala universal. En el fondo, tratamos de contrastar la hipóte- 
Emilio Delgado López-Cózar y Antonio Fernández Cano

sis de investigación que conjetura que el EC es un método fértil usado en los más diversos campos disciplinares y temáticas científicas, y que tal uso viene manteniéndose e, incluso, aumentando con el paso del tiempo. Tal evidencia podría ayudar a disipar, entonces, la infundada pero extendida creencia de que el EC es una metodología precientífica y exclusiva de determinadas ramas del conocimiento.

En la medida en que se pretende ofrecer un panorama general y universal del empleo del EC, como herramienta metodológica en las publicaciones científicas, se precisa una fuente de datos que sea multidisciplinar, en el sentido de que incluya todas las áreas de conocimiento, y, al mismo tiempo, internacional, en donde esté representada la ciencia generada en distintos países. De entre todas las fuentes de información posibles, las únicas que pueden cumplir este requisito son las bases de datos [desde ahora BD] propias del Institute for Scientific Information [desde ahora ISI] de Filadelfia; en concreto: Science Citation Index (SCI), Social Science Citation Index (SSCI) y Arts and Humanities Citation Index (A\&HCI). Estas bases, en agosto de 2001, indizaban 8648 revistas científicas ${ }^{1}$ y alrededor de 7000 libros y actas de congresos, pertenecientes en un $61 \%$ al área de Ciencia y Tecnología, el $21 \%$ al de Ciencias Sociales y un $18 \%$ a las Humanidades.

En las BD del ISI se encuentra la bibliografía científica de mayor difusión, visibilidad e impacto internacional. Es bien conocida la ley de dispersión de Bradford, la cual establece que un número muy reducido de revistas publica el mayor número de trabajos sobre un tema cualquiera, y al mismo tiempo, esas pocas revistas reciben el mayor número de citas. En concreto, según Garfield (1996), 2000 revistas publican el 85\% de los artículos y reciben el $95 \%$ de las citas. Pues bien, las BD del ISI vacían precisamente las revistas más productivas y que más influencia o impacto producen.

Por otra parte, las BD del ISI, se han convertido en una herramienta fundamental en la evaluación de las instituciones, de las revistas y de los propios científicos en buena parte del mundo, debido a la razón antedicha y a que proporcionan indicadores bibliométricos únicos (Bellavista et al. 1997; Bordons y Zulueta, 1999; Fernández Cano, 1995; Garfield y Welljams-Dorof, 1992; López-Piñero y Terrada, 1992c-d; Sancho, 1990; van Raan, 1993). Publicar en una revista indizada por el ISI se ha convertido en un signo intrínseco de calidad que es valorado positivamente por los más diversos sistemas de evaluación. Esto está provocando que las revistas cubiertas por el ISI atraigan los mejores artículos de investigación. De ahí que pueda presumirse que en las BD del ISI estén no sólo los mejores trabajos, sino también aquellos que gozan de mayor sofisti- 
El estudio de casos en las bases de datos del Science...

cación y calidad metodológica. Este último aspecto es muy relevante para el presente estudio.

En definitiva, con este trabajo se pretende conocer el uso que se viene haciendo del EC en la literatura científica internacional utilizando como espejo las BD del ISI.

\section{Material y métodos}

\subsection{Tipo de estudio}

Se trata de un estudio descriptivo longitudinal de carácter retrospectivo sobre una muestra de 49190 trabajos publicados, categorizados e indizados, como EC en las BD del SCI, SSCI y A\&HCI, entre 1992 y 2000.

\subsection{Fuente de datos}

Para la extracción de datos, se consultó la versión en CD-ROM del SCI, SSCI y A\&HCI de los años 1992 a 2000, que en la actualidad indizan 4767, 1755 y 1126 revistas, respectivamente. Los retrasos de publicación por parte de algunas revistas junto a la imposibilidad de mantener plenamente actualizadas las $\mathrm{BD}$, por lo oneroso de la carga $\mathrm{y}$ tratamiento, determina que algunos registros correspondientes al período de estudio (1992-2000) aparezcan en los CD-ROM del 2001. Es por lo que de modo complementario, se efectuaron búsquedas en los discos de dicho año (actualizaciones enero-abril para el A\&HCI y enero-julio para SCI y SSCI).

\subsection{Estrategia de búsqueda}

Dado que las BD del ISI no utilizan vocabularios temáticos controlados del tipo de los tesauros, resulta fundamental para la validez de los resultados elegir cuidadosamente los términos, los campos y la ecuación de búsqueda a implementar.

Los vocablos elegidos han sido case(s) study(ies) o case(s) report(s). Con el fin de asegurarnos de que estos son los términos habitualmente admitidos en inglés para representar el concepto objeto de estudio, se consultaron los tesauros ${ }^{2}$ empleados en las bases de datos bibliográficas de diversos campos de conocimiento. Como puede apreciarse en el Cua- 


\section{Emilio Delgado López-Cózar y Antonio Fernández Cano}

dro 1, dos son los términos empleados; predominando, tal como se anticipó, en Humanidades y Ciencias Sociales el vocablo case study-ies y en Ciencia y Tecnología case report-s. Los resultados de las búsquedas expuestos en Cuadro 1 confirman rotundamente esta aseveración.

CUADRO 1. Descriptores empleados en los tesauros de bases de datos bibliográficas internacionales para representar el concepto estudio de caso

\begin{tabular}{||l|l||}
\hline \multicolumn{1}{|c|}{ Bases de datos } & Descriptores \\
\hline Aquatic Sciences \& Fisheries Abstracts & Ninguno \\
\hline CAB International & Case reports \\
\hline Educational Resources Information Center (ERIC) & Case-studies \\
\hline Food Science and Technology Abstracts & Ninguno \\
\hline GeoRef & Case studies \\
\hline Library and Information Science Abstracts & Case studies \\
\hline MEDLINE & Case control studies \\
\hline Modern Language Association International Bibliography & Case-studies \\
\hline PSyCHINFO & Case-report \\
\hline Sociological Abstracts & Case-studies \\
\hline SPORT Discus & Case report \\
\hline The Philosopher's Index & Case study \\
\hline
\end{tabular}

La ecuación de búsqueda empleada fue: case stud $^{*}$ OR case* report ${ }^{*}$. Se utilizó, por un lado, el operador de truncamiento de sufijo (*) para recuperar el singular y plural de los vocablos elegidos y, por otro, el de adyacencia, que en las BD es implícita, pues por defecto se localizan los registros que contienen los términos adyacentes en el mismo orden y forma indicada. De este modo, se eliminan muchos documentos no pertinentes.

Los campos elegidos para la búsqueda han sido el título, resumen, descriptores o palabras-clave del autor y descriptores adicionales (Key- 
El estudio de casos en las bases de datos del Science...

word Plus). Este último es un campo que contiene palabras-clave derivadas automáticamente de los títulos de las referencias bibliográficas citadas en cada documento. No obstante, la posibilidad de buscar en todos estos campos no está disponible en las tres BD. Mientras que las versiones de SCI y SSCI lo permiten desde 1992 -y esta es la razón que justifica el ámbito cronológico elegido-, en el A\&HCI sólo es posible buscar en el título. Este inconveniente constituye una limitación importante y contribuye a sesgar los datos obtenidos en el sentido de recuperar en el A\&HCI muchos menos documentos de los potencialmente pertinentes.

El total de documentos localizados con los criterios de búsqueda expuestos fue de 52872 , de los cuales 36554 corresponden al SCI, 15287 al SSCI y 1031 al A\&HCI (véase Tabla 1). Dado que algunas revistas son indizadas simultáneamente en más de una base de datos, se procedió a detectar los duplicados, cuya cifra ascendió a 3682 documentos.

TABLA 1. Número de documentos sobre estudio de caso localizados en las bases de datos del SCI, SSCI y A\&HCI (1992-2001)

\begin{tabular}{|c|c|c|c|c|c|c|c|c|c|c|}
\hline \multirow[b]{2}{*}{ Año } & \multicolumn{3}{|c|}{ SCI } & \multicolumn{3}{|c|}{ SSCI } & \multicolumn{3}{|c|}{ A\&HCI } & \multirow[b]{2}{*}{ Total } \\
\hline & $\begin{array}{l}\text { Case* } \\
\text { Stud* }\end{array}$ & $\begin{array}{c}\text { Case } \\
\text { Report* }\end{array}$ & Total & $\begin{array}{l}\text { Case* } \\
\text { Stud* }\end{array}$ & $\begin{array}{c}\text { Case } \\
\text { Report }\end{array}$ & Total & $\begin{array}{l}\text { Case* } \\
\text { Stud* }\end{array}$ & $\begin{array}{c}\text { Case } \\
\text { Report* }\end{array}$ & Total & \\
\hline 1992 & 647 & 2420 & 3055 & 1022 & 162 & 1178 & 121 & 2 & 123 & 4356 \\
\hline 1993 & 676 & 2517 & 3189 & 1186 & 179 & 1360 & 141 & 2 & 143 & 4692 \\
\hline 1994 & 682 & 2805 & 3485 & 1134 & 181 & 1311 & 107 & 0 & 107 & 4903 \\
\hline 1995 & 748 & 3020 & 3765 & 1344 & 206 & 1538 & 115 & 1 & 116 & 5419 \\
\hline 1996 & 807 & 3364 & 4165 & 1534 & 222 & 1748 & 98 & 1 & 99 & 6012 \\
\hline 1997 & 819 & 3419 & 4235 & 1519 & 208 & 1718 & 116 & 2 & 118 & 6071 \\
\hline 1998 & 929 & 3671 & 4590 & 1722 & 228 & 1939 & 98 & 1 & 99 & 6628 \\
\hline 1999 & 1033 & 3687 & 4712 & 1778 & 203 & 1974 & 102 & 1 & 103 & 6789 \\
\hline 2000 & 1018 & 3824 & 4832 & 1951 & 244 & 2182 & 101 & 0 & 101 & 7115 \\
\hline 2001 & 157 & 369 & 526 & 331 & 8 & 339 & 22 & 0 & 22 & 887 \\
\hline Total & 7516 & 29096 & 36554 & 13521 & 1841 & 15287 & 1021 & 10 & 1031 & 52872 \\
\hline
\end{tabular}




\subsection{Variables estudiadas}

Las variables analizadas en este estudio son:

- Año de publicación, con el objeto de trazar la evolución diacrónica de la producción relativa a EC. El análisis de esa producción puede indicarnos la fertilidad, actualidad u obsolescencia del tópico metodológico que indagamos.

- Tipo de documento, para conocer el formato bibliográfico prototípico de estudios realizado bajo la metodología de EC. Se emplea la categorización utilizada por el ISI, compuesta de 28 géneros documentales.

- Campo disciplinar, a fin de conocer la importancia del EC en cada una de las áreas en que se especializa el conocimiento científico. Para ello, se utiliza la clasificación de revistas por campos científicos diseñada por el ISI que, distribuye sus casi 9000 revistas en 250 campos, de los que 168 pertenecen al SCI, 55 al SSCI y 27 al A\&HCI. A fin de obtener una visión más sintética se ha incrementado el nivel de agregación disciplinar, confeccionando una clasificación ad hoc basada en la Nomenclatura Internacional UNESCO. Se han categorizado las revistas distribuidas según campo científico ISI en 23 categorías, de las que 9 se adscriben a Ciencia y Tecnología, 7 a Ciencias Sociales y 6 a Humanidades.

El procedimiento adoptado presupone aceptar que una revista puede clasificarse simultáneamente en más de un campo (asignación múltiple) y que todos los artículos publicados en una revista pertenecen al campo temático donde la revista esté adscrita. Esto es especialmente discutible en el caso de revistas multidisciplinares que publican trabajos de muy diversas especialidades. A pesar de las posibles distorsiones que puede introducir este método de análisis, hay que decir que no existe otro que sea tan factible y operativo, y que, como ya ha señalado en otros estudios bibliométricos (Bordons y Zulueta, 1999):...toda la clasificación temática es en cierto modo arbitraria, el uso de la clasificación de revistas del ISI, que ha sido realizada con asesoramiento de expertos en cada área y está sujeta a modificaciones periódicas que garantiza su actualidad, permite una delimitación objetiva y posteriores comparaciones a lo largo del tiempo entre diversos sujetos....

- Temática asociada al EC, al objeto de denotar vínculos conceptuales entre EC y otros términos. Para ello se emplean las palabras clave asignadas por el autor o por la propia $\mathrm{BD}$ automáticamente. Es obligado advertir que 15210 registros, es decir, el 30\% de los do- 
El estudio de casos en las bases de datos del Science...

cumentos carecen de palabras clave, distribuyéndose de forma desigual en función del campo científico: Así, en Humanidades, sólo el $49 \%$ de los documentos de la muestra poseen palabras clave; mientras que en Ciencias Sociales sube al $57 \%$ y en Ciencias al 75\%. Asimismo, el promedio de palabras clave por registro es muy variable, distribuyéndose también de forma sesgada: se pasa de 5 palabras clave en Ciencias, a 3 en Ciencias Sociales y 2 en Humanidades. Esto denota que los resultados están indudablemente sesgados.

- Revistas que incluyen EC, tratando de verificar el ajuste al modelado de Bradford.

- Autoría, al objeto de determinar el número de autores que colaboran en la publicación de EC, registrando las distintas pautas de comportamiento existentes en cada disciplina. Este análisis únicamente se ha aplicado a los documentos categorizados por el ISI como originales, ya que la multiautoría puede ser sensible al género documental.

- Extensión, tratando de denotar el tamaño prototípico del artículo de EC, midiéndolo en número de páginas. Se pretende observar, al mismo tiempo, las diferencias que puedan existir entre disciplinas. Dado que la extensión de un trabajo en una revista depende del tipo documental que sea (revisión, editorial, artículo, nota...), se ha circunscrito el análisis sólo a los documentos categorizados por el ISI como originales.

\subsection{Tratamiento de los datos}

La información bibliográfica procedente de las BD del ISI se descargó en una base de datos ProCite. Los campos se exportaron al paquete informático Statistica (Statsoft 2000), para un tratamiento estadístico bastante elemental a nivel meramente descriptivo.

\section{Resultados}

\subsection{Producción diacrónica}

La producción diacrónica desde 1992 hasta 2000 para los términos de búsqueda indicados se incluye en la Tabla 2 . Es evidente que la producción ha ido, por lo general, aumentando cada año con una tasa media de crecimiento del $8 \%$. Si bien este porcentaje, ni es superior al propio de las BD del ISI, ni se ajusta a la curva de crecimiento descrita por Price 


\section{Emilio Delgado López-Cózar y Antonio Fernández Cano}

(1986), es sintomático de que estamos ante un método de investigación que no sólo no cae en desuso sino que se mantiene vivo.

TABLA 2. Trabajos publicados sobre EC en revistas indizadas en las bases de datos del SCI, SSCI y A\&HCI (1992-2000) distribuidos por tipo documental y año

\begin{tabular}{||l|c|c|c|c|c|c|c|c|c|c||}
\hline $\begin{array}{c}\text { Tipo } \\
\text { documento }\end{array}$ & 1992 & 1993 & 1994 & 1995 & 1996 & 1997 & 1998 & 1999 & 2000 & Total \\
\hline Artículo & 3351 & 2980 & 2381 & 2936 & 4963 & 4947 & 5338 & 5233 & 5749 & 37878 \\
\hline Nota & 318 & 917 & 1792 & 1718 & 14 & 0 & 0 & 0 & 0 & 4759 \\
\hline Revisión & 188 & 191 & 220 & 204 & 206 & 293 & 318 & 255 & 370 & 2245 \\
\hline Editorial & 15 & 28 & 20 & 41 & 153 & 175 & 207 & 201 & 223 & 1063 \\
\hline Actas & 59 & 86 & 57 & 134 & 137 & 154 & 172 & 214 & 237 & 1250 \\
\hline Revisión-libro & 120 & 161 & 123 & 114 & 118 & 109 & 86 & 99 & 84 & 1014 \\
\hline Carta & 63 & 79 & 87 & 97 & 79 & 87 & 124 & 103 & 109 & 828 \\
\hline Corrección & 2 & 12 & 4 & 4 & 14 & 10 & 11 & 13 & 15 & 85 \\
\hline Otros & 13 & 13 & 7 & 9 & 5 & 3 & 4 & 4 & 10 & 68 \\
\hline TOTAL & 4129 & 4467 & 4691 & 5257 & 5689 & 5778 & 6260 & 6122 & 6797 & 49190 \\
\hline \hline
\end{tabular}

\subsection{Tipo de documento}

Queda bien de manifiesto que son artículos, mayoritariamente, el tipo de documentos asociados al tópico de EC (Tabla 2); aunque habría que destacar el amplio número de revisiones y editoriales asociadas en una $\mathrm{u}$ otra medida con el EC; especialmente importante es el crecimiento a lo largo del período de estas últimas. La necesidad de editorializar y revisar periódicamente la literatura producida sobre EC podría ser un indicador de la actualidad de esta metodología. Por otro lado, destaca la radical caída de los documentos tipo «notas» sobre EC a partir de 1995, aunque estimamos que tan drástica disminución obedeciera a un cambio en la política de indización de ISI, en el sentido de asimilarlas a originales. 


\section{El estudio de casos en las bases de datos del Science...}

\subsection{Distribución por campos disciplinares}

Es de relevancia denotar como el método de EC se distribuye en los diversos campos disciplinares. La Tabla 3 da una visión diacrónica del número de EC según disciplinas.

TABLA 3. Distribución de estudios de caso publicados en revistas indizadas en SCI, SSCI y A\&HCI según disciplinas científicas (1992-2000)

\begin{tabular}{|c|c|c|c|c|c|c|c|c|c|c|}
\hline CAMPO DISCIPLINAR(*) & 1992 & 1993 & 1994 & 1995 & 1996 & 1997 & 1998 & 1999 & 2000 & Total \\
\hline Ciencia, General & 6 & 4 & 5 & 12 & 17 & 10 & 11 & 17 & 15 & 97 \\
\hline Matemáticas & 13 & 12 & 23 & 17 & 14 & 14 & 24 & 23 & 17 & 157 \\
\hline Física & 69 & 83 & 62 & 77 & 75 & 104 & 84 & 87 & 98 & 739 \\
\hline Química & 49 & 59 & 51 & 63 & 75 & 57 & 77 & 58 & 81 & 570 \\
\hline Ciencias de la Tierra y el Espacio & 81 & 99 & 89 & 100 & 120 & 143 & 158 & 174 & 166 & 1130 \\
\hline Biomedicina & 2627 & 2742 & 2961 & 3303 & 3551 & 3539 & 3891 & 3657 & 4159 & 30430 \\
\hline Ciencias Biológicas & 317 & 331 & 368 & 451 & 433 & 498 & 542 & 575 & 620 & 4135 \\
\hline Ciencias Agrarias & 78 & 65 & 69 & 79 & 70 & 111 & 118 & 87 & 87 & 764 \\
\hline Ciencias Tecnológicas & 188 & 197 & 210 & 249 & 300 & 294 & 334 & 369 & 405 & 2546 \\
\hline Ciencia y Tecnología & 3428 & 3592 & 3838 & 4351 & 4655 & 4770 & 5239 & 5047 & 5648 & 40568 \\
\hline Ciencias Sociales, General & 41 & 36 & 40 & 39 & 45 & 54 & 67 & 67 & 85 & 474 \\
\hline Ciencia política, Administración y Gestión & 154 & 170 & 180 & 201 & 256 & 242 & 238 & 279 & 351 & 2071 \\
\hline Sociología y ciencias afines & 198 & 247 & 226 & 274 & 286 & 274 & 282 & 302 & 303 & 2392 \\
\hline Ciencias de la Educación & 112 & 113 & 120 & 157 & 150 & 172 & 171 & 164 & 170 & 1329 \\
\hline Psicología y Ciencias del Comportamiento & 172 & 215 & 237 & 247 & 271 & 267 & 250 & 273 & 265 & 2197 \\
\hline Ciencias Económicas & 135 & 141 & 176 & 180 & 206 & 211 & 237 & 244 & 282 & 1812 \\
\hline Ciencias Jurídicas & 38 & 35 & 39 & 35 & 39 & 50 & 43 & 52 & 50 & 381 \\
\hline Ciencias Sociales & 850 & 957 & 1018 & 1133 & 1253 & 1270 & 1288 & 1381 & 1506 & 10656 \\
\hline Humanidades, General & 7 & 7 & 4 & 9 & 11 & 9 & 7 & 7 & 4 & 65 \\
\hline Lingüística & 34 & 37 & 47 & 36 & 52 & 38 & 35 & 54 & 58 & 391 \\
\hline Ciencias de las Artes y las Letras & 18 & 28 & 28 & 28 & 28 & 26 & 26 & 22 & 22 & 226 \\
\hline Filosofía y Teología & 13 & 18 & 11 & 10 & 18 & 22 & 18 & 28 & 20 & 158 \\
\hline Geografía & 102 & 140 & 128 & 164 & 215 & 225 & 235 & 231 & 237 & 1677 \\
\hline Historia & 41 & 32 & 34 & 41 & 44 & 40 & 55 & 43 & 42 & 372 \\
\hline Humanidades & 215 & 262 & 252 & 288 & 368 & 360 & 376 & 385 & 383 & 2889 \\
\hline
\end{tabular}

(*) Según clasificación Nomenclatura Internacional de la UNESCO 
La presencia de EC en las más diversas disciplinas y áreas de conocimiento es palmaria, aunque son evidentes también grandes diferencias en número de documentos relativos a EC según campo general. No sorprende, que sea en Ciencia y Tecnología donde más se use el EC (75\% de los documentos), frente a las Ciencias Sociales (19,7\%) y Humanidades $(5,3 \%)$, aunque sí las diferencias porcentuales tan acusadas que se producen. Lo más destacable es el elevado valor alcanzado en Ciencia y Tecnología, muy por encima de lo que representa esta área en las BD del ISI (61\%), y el ínfimo obtenido por las Humanidades, muy por debajo de lo que representa el área en las BD del ISI (18\%). Resulta paradójica la escasa popularidad en Humanidades de un método con una fuerte tradición histórico-humanista (véanse aportes ad hoc en este mismo número), plasmado en autobiografías, historias de vida o episodios vitales. Esta omisión pudiera ser imputable más a los sesgos de que adolece este trabajo que a una escasa preocupación metodológica por el EC por parte de artistas y «gentes de letras». Las peores prestaciones en recuperación de la información que presenta el A\&HCI, donde sólo es posible buscar en el título y no en el resumen y palabras clave que, por otra parte, escasean en esta base de datos, junto a la nula estandarización, el bajo consenso cognitivo y metodológico existente en las áreas humanísticas y a su diversidad terminológica, pues podrían ser múltiples los vocablos con que fuera conocido el EC (biografías, historias de vida...), es lo que explicaría estos paupérrimos porcentajes. Los mismos argumentos, pero vueltos del revés, podrían explicar los buenos resultados del EC en Ciencia y Tecnología. Las Ciencias Sociales, en cambio, se posicionarían en una situación de «normalidad».

Si acercamos más la lente del retrato disciplinar, recurriendo a la clasificación por áreas temáticas empleada por el ISI, podremos detectar cuales serían las disciplinas concretas (especialidades utilizando la terminología biosanitaria) más productivas en EC. Una relación de las 30 áreas más productivas, en cuanto a número de documentos que guardan cierta relación con EC, viene dada por la Tabla 4. De esas 30 primeras áreas, todas corresponden a especialidades biomédicas excepto cinco, de las que cuatro se adscriben a las Ciencias Sociales (Educación e Investigación Educativa, Planificación y Desarrollo, Gestión, Estudios Medioambientales) y una al área biológica. 


\section{El estudio de casos en las bases de datos del Science...}

TABLA 4. Escalafón de las treinta disciplinas específicas que incorporan un mayor número de documentos relativos estudio de caso

\begin{tabular}{|c|c|c|c|c|}
\hline $\mathrm{R}^{\mathrm{o}}$ & Área o disciplina específica & \# Documentos (A) & \#Revistas (B) & Razón A/B \\
\hline $1^{\mathrm{a}}$ & Cirugía & 5770 & 51 & 113.1 \\
\hline $2^{\mathrm{a}}$ & Neurología Clínica & 4280 & 54 & 79.3 \\
\hline $3^{\mathrm{a}}$ & Pediatría & 1827 & 34 & 53.7 \\
\hline $4^{\mathrm{a}}$ & Medicina General e Interna & 1790 & 42 & 42.6 \\
\hline $5^{\mathrm{a}}$ & Patología & 1782 & 30 & 59.4 \\
\hline $6^{\mathrm{a}}$ & Obstetricia y Ginecología & 1452 & 18 & 80.7 \\
\hline $7^{\mathrm{a}}$ & Oncología & 1439 & 33 & 43.6 \\
\hline $8^{\mathrm{a}}$ & Radiología, Medicina Nuclear y... & 1391 & 23 & 60.5 \\
\hline $9^{\mathrm{a}}$ & Estomatología, Cirugía Oral y... & 1336 & 24 & 55.7 \\
\hline $10^{\mathrm{a}}$ & Dermatología y E. Venéreas & 1276 & 20 & 63.8 \\
\hline $11^{\mathrm{a}}$ & Psiquiatría & 1265 & 48 & 26.4 \\
\hline $12^{\mathrm{a}}$ & Gastroenterología y Hepatología & 1018 & 19 & 53.6 \\
\hline $13^{\mathrm{a}}$ & Sistema Cardíaco y Cardiovascular & 859 & 22 & 39.0 \\
\hline $14^{\mathrm{a}}$ & Oftalmología & 847 & 18 & 47.1 \\
\hline $15^{\mathrm{a}}$ & Enfermedad Vascular Periférica & 842 & 11 & 76.5 \\
\hline $16^{\mathrm{a}}$ & Inmunología & 828 & 13 & 63.7 \\
\hline $17^{\mathrm{a}}$ & Hematología & 782 & 17 & 46.0 \\
\hline $18^{\mathrm{a}}$ & Enfermedades Infecciosas & 758 & 16 & 47.4 \\
\hline $19^{\mathrm{a}}$ & Educación e Investigación Educativa & 751 & 37 & 20.3 \\
\hline $20^{\mathrm{a}}$ & Otorrinolaringología & 711 & 11 & 64.6 \\
\hline $21^{\mathrm{a}}$ & Ortopedia & 683 & 7 & 97,6 \\
\hline $22^{\mathrm{a}}$ & Neurociencias & 682 & 34 & 20,1 \\
\hline $23^{\mathrm{a}}$ & Urología y Nefrología & 662 & 15 & 44,1 \\
\hline $24^{\mathrm{a}}$ & Planificación y Desarrollo & 660 & 25 & 26,4 \\
\hline $25^{\mathrm{a}}$ & Gestión & 654 & 24 & 27,3 \\
\hline $26^{\mathrm{a}}$ & Farmacia y Farmacología & 628 & 24 & 26,2 \\
\hline $27^{\mathrm{a}}$ & Ciencias Medioambientales & 610 & 24 & 25,4 \\
\hline $28^{\mathrm{a}}$ & Estudios Medioambientales & 585 & 22 & 26,6 \\
\hline $29^{\mathrm{a}}$ & Genética y Herencia & 520 & 10 & 52,0 \\
\hline $30^{\mathrm{a}}$ & Biología Celular & 488 & 5 & 97,6 \\
\hline
\end{tabular}




\section{Emilio Delgado López-Cózar y Antonio Fernández Cano}

La razón $\mathrm{A} / \mathrm{B}$ (número de revistas / número de documentos recuperados) podría ser un indicador del grado de uso del EC dentro de cada disciplina. Es de resaltar que, en la especialidad Medicina de los Cuidados Clínicos (Clinical Care Medicine), esa razón alcanza el máximo valor 201.5 (403 documentos / 2 revistas).

El empleo del EC en las ciencias de la salud, en general, y en algunas de sus revistas más prestigiosas tanto a escala internacional (The New England Journal of Medicine o The Lancet) como en el caso español (Medicina Clínica o Revista Clínica Española) había sido evidenciado hace años (Álvarez Dardet et al. 1987). No obstante, se ha detectado un descenso considerable en el empleo de dicha metodología en estas revistas: ha pasado, de ser empleada en el $30 \%$ de los originales publicados en dichas revistas en los setenta, a un exiguo 5\% en los noventa (Aibar et al. 1999). Estos datos no concuerdan con lo reflejado en este estudio, aunque bien pudiera ocurrir que dicho descenso se produzca en las revistas de primer nivel y no en el conjunto. Asimismo, por otra parte, Pardo García et al. (1996) destacaron la importancia del EC en Cirugía, que es la especialidad que se muestra más productiva en esta metodología.

\subsection{Análisis temático}

Bajo este epígrafe tratamos de relacionar términos presentes en un mismo documento asociados a nuestros términos de búsqueda (case study o case report). En concreto, hemos recuperado 65536 palabras clave ligadas a esos términos. El Cuadro 2 reproduce las treinta más frecuentes y el número de veces (\#) en que tal asociación dual se presenta.

CUADRo 2. Términos asociados a estudio de caso en las bases de datos del SCI, SSCI y A\&HCI, y cantidad de asociaciones (1992-2000)

\begin{tabular}{||l|l|l||}
\hline \multicolumn{2}{|c||}{ Rango/Término (\#) } \\
\hline $1^{\circ}$ Niños (970) & $11^{\circ}$ Preñez (374) & $21^{\circ}$ Infancia (290) \\
$2^{\circ}$ Gestión (957) & $12^{\circ}$ Cirugía (339) & $22^{\circ}$ Informe (288) \\
$3^{\circ}$ Diagnóstico (939) & $13^{\circ} \mathrm{MRI}^{3}(327)$ & $23^{\circ}$ Rasgos (265) \\
$4^{\circ}$ Enfermedad (881) & $14^{\circ} \mathrm{CT}^{4}(324)$ & $24^{\circ}$ Lesiones (260) \\
$5^{\circ}$ Terapia (881) & $15^{\circ}$ AIDS $^{\circ}(322)$ & $25^{\circ}$ Cerebro (259) \\
$6^{\circ}$ Paciente (533) & $16^{\circ}$ Experiencia (312) & $26^{\circ}$ Complicaciones (254) \\
$7^{\circ}$ Carcinoma (522) & $17^{\circ}$ Quimioterapia (306) & $27^{\circ}$ Tumor (249) \\
$8^{\circ}$ Infección (503) & $18^{\circ}$ Expresión (303) & $28^{\circ}$ Inmunohistoquímica (247) \\
$9^{\circ}$ Cáncer (476) & $19^{\circ}$ Riesgo (301) & $29^{\circ}$ Gen (244) \\
$10^{\circ}$ Modelo (416) & $20^{\circ}$ Infante (292) & $30^{\circ}$ Células (236) \\
\hline \hline
\end{tabular}


El estudio de casos en las bases de datos del Science...

Los términos más frecuentemente asociados a EC son susceptibles de agruparse en tres categorías: el mundo de los niños (niños, infante, infancia,..), la relación salud-enfermedad (enfermedad, paciente, carcinoma, infección,...) y aspectos operativos (gestión, diagnóstico, experiencia, informe,...). Es evidente que la temática biosanitaria represente la parte central de la relación establecida.

\subsection{Distribución según revistas}

En esta radiografía intensiva y extensiva del ISI, también hemos localizado cuáles y cuántas revistas, incluidas en tales $\mathrm{BD}$, publican artículos relativos a EC. Se han localizado un total de 4689 (casi dos tercios) revistas que incorporan EC; una sola de ellas (Neurosurgery) incluye 1387 documentos afines al método que aquí se indaga.

Cuando relacionamos número de revistas con número de documentos afines a EC, denotamos un patrón de resultados propio del modelado de Bradford (1948); en el sentido de que unas pocas revistas concentran la inmensa mayoría de los documentos sobre EC; véase tabla 5:

TABLA 5. Relación de número de documentos y de revistas indizadas en las bases de datos del SCI, SSCI Y A\&HCI (1992-2000)

\begin{tabular}{||l|c|c|c|c|c|c|c|c||}
\hline \hline \# documentos & $>1000$ & $600-501$ & $500-401$ & $400-301$ & $300-201$ & $200-101$ & $100-91$ & $90-81$ \\
\hline \# revistas & 1 & 2 & 2 & 5 & 11 & 34 & 16 & 37 \\
\hline \# documentos & $80-71$ & $70-61$ & $60-51$ & $50-41$ & $40-31$ & $30-21$ & $20-11$ & $10-1$ \\
\hline \# revistas & 22 & 18 & 40 & 66 & 97 & 170 & 493 & 3675 \\
\hline
\end{tabular}

Las veinte primeras revistas (un escaso 5 por mil) incorporan ya 7659 documentos relativos a $\mathrm{EC}$ (el $14.5 \%$ del total); véase tabla 6 , junto con la ordenación correspondiente. Las primeras 521 revistas $(11.1 \%)$ agrupan 27102 documentos (51.2 \%). Por ello, aunque el ajuste al modelo de desarrollo geométrico que propuso Bradford (1948) no sea tajante, sí es evidente un patrón de acomodación a dicho modelado. 


\section{Emilio Delgado López-Cózar y Antonio Fernández Cano}

TABLA 6. Relación de la 20 revistas indizadas en las bases de datos del SCI, SSCI Y A\&HCI que publican más estudios de casos (1992-2000)

\begin{tabular}{|r|l|c||}
\hline $\mathrm{R}^{\circ}$ & \multicolumn{1}{|c|}{ Revista } & \# Documentos \\
\hline $1^{\circ}$ & Neurosurgery & 1387 \\
\hline $2^{\circ}$ & Surgical Neurology & 560 \\
\hline $3^{\circ}$ & Journal of Neurosurgery & 535 \\
\hline $4^{\circ}$ & Journal of Reproductive Medicine & 463 \\
\hline $5^{\circ}$ & Angiology & 409 \\
\hline $6^{\circ}$ & Acta Cytologica & 393 \\
\hline $7^{\circ}$ & Clinical Infections Deseases & 387 \\
\hline $8^{\circ}$ & Annales de Dermatologie et de Venereologie & 368 \\
\hline $9^{\circ}$ & American Journal of Ophtalmology & 322 \\
\hline $10^{\circ}$ & Archive de Pediatrie & 307 \\
\hline $11^{\circ}$ & Presses Medicales & 307 \\
\hline $12^{\circ}$ & Annals of Pharmacotherapy & 240 \\
\hline $13^{\circ}$ & Journal of Laringology and Otology & 239 \\
\hline $14^{\circ}$ & Journal of Bone and Joint Surgery-American Volume & 237 \\
\hline $15^{\circ}$ & Journal of Oral and Maxilofacial Surgery & 227 \\
\hline $16^{\circ}$ & Clinical Radiology & 224 \\
\hline $17^{\circ}$ & Cancer & 222 \\
\hline $18^{\circ}$ & Journal of Trauma-Injury Infection and... & 222 \\
\hline $19^{\circ}$ & Skeletal Radiology & 221 \\
\hline $20^{\circ}$ & Journal of Urology & \\
\hline \hline
\end{tabular}

Ajustándose a la composición de las BD del ISI, las revistas norteamericanas y en lengua inglesa son las más abundantes, aunque para esta rełación es bastante destacable el número de revistas francesas: Annales de Dermatologie et de Venereologie, Archive de Pediatrie y Presses Medicales.

Es también llamativo que sean tres revistas de Neurocirugía las que encabecen la lista de publicaciones más productivas. Son, además, las responsables directas de que tanto la Cirugía como la Neurología Clínica se erijan en las especialidades que mejor abono ofrecen para cultivar la metodología del EC. 
El estudio de casos en las bases de datos del Science...

\subsection{Autoría}

La propia naturaleza del EC, junto al hecho de que sea un método que presenta una menor complejidad metodológica, unas menores exigencias en la recogida, tratamiento y análisis de datos, $\mathrm{y}$, por ende, una menor carga de trabajo global, debieran traducirse en una exigua tasa de colaboración o multiautoría. Pues bien, muy en contra de lo que pudiera pensarse, el número de autores que firman las publicaciones de EC es muy elevado, puesto que el 55,5\% de los documentos poseen entre dos y cuatro autores, el $22,5 \%$ cinco o más, y sólo el $21 \%$ tienen un autor. Se ha llegado incluso a encontrar cuatro trabajos con más de 51 autores. Sin embargo, esta distribución no es nada uniforme ya que existen profundas divergencias entre campos científicos. Así, mientras que en Ciencia y Tecnología, el promedio de autores por trabajo es superior a tres y el autor único está en peligro de extinción (11\% de los trabajos); en Humanidades, el promedio se sitúa en un autor que es, por otra parte, la especie mayoritaria (60\% de los trabajos); y en Ciencias Sociales el promedio de firmas por trabajo se sitúa en dos y el autor único representa algo menos de la mitad (47\%). Se aprecia una perfecta gradación entre las ciencias «duras», cuyo núcleo está constituido por las ciencias de la vida y las biomédicas, en particular, y las «blandas», conformadas por las Artes y las Letras donde es el trabajo individual es el que prima. Este patrón no es más que una nueva manifestación de unos hábitos de publicación, que son bien conocidos, que han sido prolijamente descritos y que responde, en parte, a la presión por publicar que recorre como reguero de pólvora todos los círculos científicos. 


\section{Emilio Delgado López-Cózar y Antonio Fernández Cano}

TABLA 7. Número de autores en los artículos originales sobre estudio de caso publicados en revistas indizadas en las bases de datos del SCI, SSCI Y A\&HCI (1992-2000) .

\begin{tabular}{|c|c|c|c|c|c|c|c|c|c|c|}
\hline \multirow{2}{*}{ CAMPO DISCIPLINAR } & \multirow{2}{*}{$\begin{array}{c}\mathrm{N}^{0} \text { artículos } \\
\text { originales }\end{array}$} & \multirow{2}{*}{ Media \pm DS } & \multirow{2}{*}{ Moda } & \multicolumn{7}{|c|}{ NÚMERO DE AUTORES } \\
\hline & & & & 1 & $2 \mathrm{a} 4$ & 5 a 9 & 10 a 15 & 16 a 25 & $26 a 50$ & $>51$ \\
\hline Ciencia, General & 84 & $3,2 \pm 3,0$ & 1 & 26 & 41 & 13 & 3 & 1 & & \\
\hline Matemáticas & 151 & $2,1 \pm 1,1$ & 2 & 49 & 98 & 4 & & & & \\
\hline Física & 708 & $2,9 \pm 1,9$ & 2 & 129 & 482 & 89 & 6 & 1 & & \\
\hline Química & 409 & $3,2 \pm 1,6$ & 3 & 37 & 287 & 84 & 1 & & & \\
\hline Ciencias de la Tierra y el Espacio & 1076 & $3,3 \pm 3,0$ & 2 & 185 & 693 & 165 & 27 & 4 & 1 & 1 \\
\hline Biomedicina & 21772 & $4,0 \pm 2,3$ & 3 & 1868 & 12386 & 7076 & 407 & 18 & 5 & 3 \\
\hline Ciencias Biológicas & 3326 & $3,6 \pm 2,4$ & 2 & 529 & 1890 & 834 & 65 & 6 & 2 & \\
\hline Ciencias Agrarias & 617 & $3,0 \pm 1,7$ & 2 & 95 & 419 & 98 & 5 & & & \\
\hline Ciencias Tecnológicas & 2412 & $2,6 \pm 1,5$ & 2 & 555 & 1647 & 199 & 7 & 1 & & \\
\hline Ciencia y Tecnología & & $3,1 \pm 2,1$ & 2 & 3474 & 17943 & 8562 & 521 & 31 & 8 & 4 \\
\hline Ciencias Sociales, General & 451 & $1,8 \pm 1,1$ & 1 & 231 & 207 & 11 & & & & \\
\hline Ciencia Política, Administración y .. & 1901 & $1,8 \pm 1,1$ & 1 & 858 & 1011 & 30 & 2 & & & \\
\hline Sociología y ciencias afines & 2088 & $1,7 \pm 1,1$ & 1 & 1191 & 858 & 35 & 1 & & & \\
\hline Ciencias de la Educación & 1328 & $1,9 \pm 1,3$ & 1 & 594 & 579 & 49 & 1 & & & \\
\hline Psicología y CC. del Comportamiento & 1657 & $2,4 \pm 1,5$ & 1 & 548 & 954 & 152 & 3 & & & \\
\hline Ciencias Económicas & 1636 & $1,8 \pm 1,1$ & 1 & 797 & 816 & 20 & 3 & & & \\
\hline Ciencias Jurídicas & 318 & $1,8 \pm 1,2$ & 1 & 169 & 139 & 8 & 1 & & & \\
\hline Ciencias Sociales & & $1,9 \pm 1,2$ & 1 & 4388 & 4564 & 305 & 11 & & & \\
\hline Humanidades, General & 65 & $1,0 \pm 0,4$ & 1 & 57 & 5 & & & & & \\
\hline Lingüística & 287 & $1,8 \pm 1,2$ & 1 & 155 & 122 & 10 & & & & \\
\hline Ciencias de las Artes y las Letras & 159 & $1,2 \pm 0,5$ & 1 & 125 & 24 & & & & & \\
\hline Filosofía y Teología & 135 & $1,4 \pm 0,7$ & 1 & 93 & 40 & 1 & & & & \\
\hline Geografia & 1492 & $1,7 \pm 1,0$ & 1 & 783 & 685 & 22 & 2 & & & \\
\hline Historia & 251 & $1,3 \pm 0,9$ & 1 & 206 & 40 & 4 & & & & \\
\hline Humanidades & & $1,4 \pm 0,8$ & 1 & 1419 & 916 & 37 & 2 & & & \\
\hline
\end{tabular}

\subsection{Extensión}

La diversidad de formatos, diseños tipográficos, estilos, familias y cuerpos de los caracteres empleados, en las casi cinco mil revistas objeto de este estudio, hacen muy difícil, por no decir casi imposible, definir el tamaño prototípico del artículo original sobre EC. Sin embargo, sí se puede afirmar que su extensión no es reducida (en torno a 13 páginas), comparada con la de otros tipos de artículos. Bien es verdad que se denotan grandes diferencias entre los artículos provenientes de las distintas disciplinas. Así, los artículos de revistas de Ciencia y Tecnología ocu- 
pan la mitad de espacio ( 7 páginas es lo habitual) que los de Ciencias Sociales, que son ligeramente más cortos (Moda 12, Mediana 13) que los de Humanidades (Moda 15, Mediana 14). De nuevo se observa una perfecta modulación según el grado de «dureza» del campo disciplinar: se va de 4 páginas de promedio en Biomedicina hasta 20 en Historia.

TABLA 8. Extensión en páginas de los artículos originales sobre estudio de caso publicados en revistas indizadas en las bases de datos del SCI, SSCI Y A\&HCI (1992-2000)

\begin{tabular}{||l|c|c|c|c||}
\hline \multirow{2}{*}{ CAMPO DISCIPLINAR } & \multicolumn{3}{|c||}{ NÚMERO DE PÁGINAS } \\
\cline { 2 - 5 } & $\begin{array}{c}\text { No }^{0} \text { artículos } \\
\text { originales }\end{array}$ & Media \pm DS & Moda & Mediana \\
\hline Ciencia, General & 84 & $6,8 \pm 9$ & 4 & 5 \\
Matemáticas & 151 & $16,5 \pm 9,3$ & 12 & 15 \\
Física & 708 & $10,4 \pm 7,7$ & 7 & 8 \\
Química & 409 & $8,3 \pm 4,8$ & 6 & 7 \\
Ciencias de la Tierra y el Espacio & 1076 & $13,1 \pm 6,6$ & 7 & 12 \\
Biomedicina & 21772 & $4,2 \pm 3,6$ & 2 & 3 \\
Ciencias Biológicas & 3326 & $7,8 \pm 6,1$ & 3 & 6 \\
Ciencias Agrarias & 617 & $7,9 \pm 5,5$ & 3 & 7 \\
Ciencias Tecnológicas & 2412 & $11 \pm 7,2$ & 6 & 10 \\
Ciencia y Tecnología & & $9,6 \pm 6,6$ & 7 & 7 \\
Ciencias Sociales, General & 451 & $16,6 \pm 8,0$ & 12 & 15 \\
Ciencia política, Administración y Gestión & 1901 & $15,7 \pm 7,8$ & 11 & 14 \\
Sociología y ciencias afines & 2088 & $16,0 \pm 8,1$ & 15 & 15 \\
Ciencias de la Educación & 1328 & $14,7 \pm 12,8$ & 12 & 13 \\
Psicología y Ciencias del Comportamiento & 1657 & $12,2 \pm 8,1$ & 8 & 10 \\
Ciencias Económicas & 1636 & $15,6 \pm 7,7$ & 12 & 14 \\
Ciencias Jurídicas & 318 & $19,1 \pm 16,9$ & 16 & 16 \\
Ciencias Sociales & & $15,7 \pm 9,9$ & 12 & 14 \\
Humanidades, General & 65 & $12,5 \pm 11,9$ & 1 & 11 \\
Lingǘstica & 287 & $18,7 \pm 9,8$ & 15 & 17 \\
Ciencias de las Artes y las Letras & 159 & $13,7 \pm 9,8$ & 6 & 12 \\
Filosofía y Teología & 135 & $13,5 \pm 7,9$ & 19 & 13 \\
Geografía & 1492 & $15,8 \pm 7,6$ & 15 & 15 \\
Historia & 251 & $20,2 \pm 10,7$ & 15 & 19 \\
Humanidades & & $15,7 \pm 9,6$ & 15 & 14 \\
\hline
\end{tabular}




\section{Emilio Delgado López-Cózar y Antonio Fernández Cano}

\section{Discusión y conclusiones}

La solvencia de los resultados arrojados por un estudio, como el que aquí se presenta, depende de que la fuente de datos elegida cubra adecuadamente el tópico objeto de análisis. Los sesgos temáticos, geográficos y lingüísticos que poseen las BD del ISI han sido comentados reiteradamente (Braun et al. 2000; Fernández Cano, 1999; Gibbs, 1995; Gómez y Bordons, 1996; López-Piñero y Terrada 1992a-b; Spinak, 1996). Entre ellos cabe destacar los siguientes:

1. Sesgo a favor de la ciencia básica, en general, y hacia disciplinas como la Física, Química, Matemáticas, Biología y Farmacología. En particular, las Ciencias Médicas que se encuentran ampliamente cubiertas en detrimento de las áreas aplicadas y tecnológicas (Ingeniería, Arquitectura, Agricultura, Informática, Metalurgia...), que están infrarrepresentadas (Braun et al. 2000). Esto quiere decir, que los temas de interés básico y alcance internacional están mejor reflejados que los de interés local y alcance local o regional.

2. Sesgo a favor de la ciencia producida en los países anglosajones. Las revistas publicadas en países como Suiza, Holanda, Reino Unido y países escandinavos están sobre-representadas frente a las editadas en países de la periferia científica y, especialmente, de las provenientes del Tercer Mundo (Gibbs, 1995). El potencial editorial de estos países en las BD del ISI está muy por encima de su potencial editorial real (Braun et al. 2000). Ejemplo paradigmático es, de un lado, Reino Unido y, de otro, España, que posee 44 revistas en el conjunto de las BD del ISI. Este exiguo número representa el $0,5 \%$ del total, un valor muy por debajo de su potencial científico y editorial, que viene calculándose en todos los indicadores de producción científica torno a valores del 2,5-3\%.

3. Sesgo a favor de las publicaciones en lengua inglesa. En 1997, el 95\% de los artículos indizados en el SCI eran publicados en inglés (Garfield, 1998).

A pesar de que la cobertura de las $\mathrm{BD}$ va sufriendo pequeñas variaciones (en torno a 250 revistas se incorporan anualmente), los sesgos perduran con el paso del tiempo debido a los criterios empleados en la selección de revistas (Garfield, 1990; Testa 1998). El factor clave para la inclusión de una revista es su tasa de citación. Son las revistas de orientación básica, publicadas en países anglosajones y en lengua inglesa las que más probabilidad tienen de ser citadas por las revistas que ya se encuentran indizadas que, son a su vez, muy representativas de la comunidad anglosajona. 
El estudio de casos en las bases de datos del Science...

Ahora bien, aun siendo verdad todo lo comentado, de lo que no hay duda es que en las BD del ISI se encuentra la bibliografía científica de mayor difusión, visibilidad, impacto internacional y sofisticación metodológica.

Por otra parte, siempre nos quedará una duda razonable sobre si, al efectuarse la búsqueda en lenguaje natural, los términos elegidos cubren la multiplicidad de vocablos con que es conocida la metodología de $\mathrm{EC}$, en las distintas áreas de conocimiento e indizada en las BD que las representan. En este sentido, conviene recordar el importante sesgo detectado en el trabajo que penaliza los resultados hallados en las Humanidades.

Sin embargo, bien podemos afirmar que la hipótesis que anticipamos se verifica plenamente: el EC como método de investigación es usado en los más diversos campos disciplinares, disciplinas y temáticas; con un uso que se ha venido acrecentando progresivamente. Un campo disciplinar cual es el biosanitario acapara la mayoría de los EC indizados en ISI. No parece entonces justificada la creencia que asocia EC con la preciencia o con las Humanidades, sino, que se nos manifiesta como un rico y fértil método de indagación, que opera en todo los ámbitos del conocimiento.

Más insidioso sería aún imputar a las áreas o especialidades, donde se realizan estudios con metodología de EC, un estatus científico bajo, en las que el conocimiento no es bastante consistente, universal ni bien articulado. Antes bien, casos específicos y relevantes siempre estarán presentes en la realidad natural, social y cultural; cuan más compleja sea esa realidad y más ansia tengamos de conocer, se nos manifestaran casos, cuyo estudio constituye el primer paso básico del quehacer científico.

\section{Notas}

${ }^{1}$ Se realizó la consulta al Master Journal List del ISI el 29-08-2001; disponible en red: http://www.isinet.com/cgi-bin/jrnlst/jlresults,cgi?PC=MASTER.

${ }^{2}$ Los tesauros son herramientas terminológicas que determinan cuales son las palabras admitidas y no admitidas para describir un concepto, estableciendo relaciones jerárquicas y de correspondencia entre ellas. Su uso generalizado, en la recuperación de información y su aplicación por parte de especialistas en cada área temática, asegura la pertinencia de los términos elegidos.

${ }^{3}$ MRI: Magnetic Resonance Imaging (Imágenes por resonancia magnética).

${ }^{4}$ CT: Computer Tomography (Tomografía por ordenador).

${ }^{5}$ AIDS: SIDA. 


\section{Emilio Delgado López-Cózar y Antonio Fernández Cano}

\section{Bibliografía}

Aibar Remón, C.; Rabanaque, M. a J.; Álvarez Dardet, C.; Nolasco, A. y Moncho, J.; Gascón, E. (1999): Evolución de los diseños epidemiológicos de la investigación clínica española (1975-1994). Revista Española de Salud Pública 73, 445-453.

Álvarez Dardet, C.; Mur, P.; Gascón, E.; Nolasco, A. y BolúMar, F. (1987): La investigación clínica en España: Tipos de diseños utilizados. Medicina Clínica 89, 221-223

Bellavista, J.; Guardiola, E.; Méndez, A. y Bordons, M. (1997): Evaluación de la investigación. Madrid: Centro de Investigaciones Sociológicas.

Bordons, M. y ZuLuETA, M. A. (1999): Evaluación de la actividad científica a través de indicadores bibliométricos. Revista Española de Cardiología 52, 790-800.

BradFord, S. C. (1948): Documentation. Londres: Crossby Lockwood. Reimpresión en (1950) Washington, D.C.: Public Affair Press.

Braun, T.; Glänzel, W. y Schubert, A. (2000): How balanced is the Science Citation Index's journal coverage? A preliminary overview of macrolevel statistical data. En The web of knowledge: A festschrift in honor of Eugene Garfield, B. Cronin y H. Barsky (Eds.) (pp. 251-271). ASIS\&T Monograph Series.

Fernández CANo, A. (1995): Métodos para evaluar la investigación en Psicopedagogía. Madrid: Síntesis.

FERnÁNDeZ CANo, A. (1999): Producción educativa española en el Social Sciences Citation Index (1988-1997). Revista Española de Pedagogía 214, 509-524.

GARFIELD, E. (1979): Citation indexing: Its theory and application in science, technology and humanities. John Wiley \& Sons, Nueva York. Reimpresión (1983), Filadelfia: ISI Press.

GARFIELD, E. (1990): How ISI selects journals for coverage: Quantitative and qualitative considerations. Current Contents 28(22), 5-13.

GARFIELD, E. (1996): The significant scientific literature appears in a small core of journals. The Scientist 10(17), 13-16.

GARFIELD, E. (1998): Mapping the world of science. En Topical paper presented at the 150th Anniversary Meeting of the AAAS. Filadelfia. Accesible en http://www.garfield.library.upenn.edu/papers/mapsciworld.html [Consultado 29-agosto-2001]

GARFIELD, E. y Welluams Dorof, A. (1992): Citation data: Their use as quantitative indicators for science and technology evaluation and policy-making. Science and Public Policy 19, 321-327.

GiBBS, W. W. (1995): Lost science in the Third World. Scientific American, August, 92-99,

Gómez, I. y Bordons, M. (1996): Limitaciones en el uso de los indicadores bibliométricos para la evaluación científica. Política Científica 46, 21-26.

López Piñero, J. M. y Terrada, M. L. (1992a): Los indicadores bibliométricos y la evaluación de la actividad médico científica (I). Usos y abusos de la bibliometría. Medicina Clínica 98(2), 64-68.

López Piñero, J. M. y Terrada, M. L. (1992b): Los indicadores bibliométricos y la evaluación de la actividad médico científica (II). La comunicación científica en las distintas áreas de las ciencias médicas. Medicina Clínica 98(3), 101-106.

López Piñero, J. M. y Terrada, M. L. (1992c): Los indicadores bibliométricos y la evaluación de la actividad médico científica (III). Los indicadores de producción, circulación y dispersión, consumo de la información y repercusión. Medicina Clinica 98(4), 142-148. 


\section{El estudio de casos en las bases de datos del Science...}

López PiÑero, J. M. y Terrada, M. L. (1992d): Los indicadores bibliométricos y la evaluación de la actividad médico-científica (IV). La aplicación de los indicadores. Medicina Clínica 98(10), 384-388.

Price, D. J. de S. (1986): Little Science, big science... and beyond. Nueva York: Columbia University Press.

SANCHO, R. (1990): Indicadores bibliométricos utilizados en la evaluación de la ciencia y la tecnología. Revisión bibliográfica. Revista Española de Documentación Científica 13, 842-865.

SPINAK, E. (1996): Diccionario enciclopédico de bibliometría, cienciometría e informetría. Caracas: UNESCO.

StATSFOT (2000): Statistica for Windows. Computer program manual. Tulsa: Statsoft Incorporated.

TESTA, J. (1998): The ISI Database: The Journal Selection Process. Accesible en: http:// www.isinet.com/isi/hot/essays/selectionofmaterialforcoverage/199701.html [Consultado 2001-10-07]

VAN RAAN, A. F. J. (1993): Advanced bibliometric methods to assess research performance and scientific development: Basic principles and recent practical applications. $R e$ search Evaluation 3, 151-166. 